\title{
Implante dental inmediato en paciente diabético
}

\section{Immediate dental implant post-extraction on systemically compromised patient}

\author{
Isabella Manzur Villalobos ${ }^{1 *}$, María Alejandra Puerta Domínguez ${ }^{1}$, Sandra Blanco Prado², \\ Guillermo Machuca Portillo³, Antonio Díaz Caballero ${ }^{4}$
}

1. Odontóloga Universidad de Cartagena. Grupo de Investigaciones GITOUC Universidad de Cartagena.

2. Odontóloga. Magíster en Odontología Integrada de Adulto y Pacientes Especiales- Universidad de Sevilla.

3. Odontólogo. Profesor titular de Clínica

Odontológica Integrada de Pacientes Especiales.

Director del Master de Odontología Integrada

de Adultos y Pacientes Especiales, Facultad de

Odontología- Universidad de Sevilla.

4. Odontólogo Universidad de Cartagena.

Especialista en Periodoncia Universidad Javeriana,

Magíster en Educación Universidad del Norte.

Doctor en Ciencias Biomédicas Universidad de

Cartagena. Profesor titular Facultad de Odontología

Universidad de Cartagena.

* Correspondencia autor. Isabella Manzur Villalobos. Facultad de Odontología Universidad de Cartagena. Campus de la Salud Zaragocilla. Cartagena, Bolívar, Colombia, Sur América. Teléfono (057+) (05) 6698172 - 6698173 Ext. 110. Fax Ext. 124

Correo electrónico: imanzurv@unicartagena.edu.co Universidad de Sevilla, Sevilla, España.

Trabajo recibido el 28/01/2016. Aprobado para su publicación el 08/02/2017

\begin{abstract}
RESUMEN
Actualmente el implante dental se considera una buena alternativa para reemplazar los órganos dentarios faltantes. Sin embargo, existen ciertos requerimientos necesarios para la colocación de este y obtener una buena resolución. Estudios actuales demuestran que la colocación inmediata del implante dental posterior a la extracción dental obtiene mejores resultados a largo plazo y preserva el espacio alveolo dental natural por lo cual facilita la inserción y correcto ajuste del implante dental, siempre y cuando la extracción sea atraumática y sin complicaciones. Por ende, en el presente caso se reporta paciente masculino de 73 años con antecedentes de hipertensión arterial y diabetes mellitus tipo II, controlado con su médico especialista, al cual se le realizó extracción dentaría atraumática de OD\#46 con inmediata colocación de implante dental NORMON HI e inserción de xenoinjerto óseo BIO-GEN para asegurar correcta oseointegración, cuyo procedimiento se realizó sin complicaciones y con correcta posición y paralelismo.
\end{abstract}

PALABRAS CLAVE

Implantes dentales, diabetes mellitus, enfermedades sistémicas, extracción dental.

Rev. Clin. Periodoncia Implantol. Rehabil. Oral Vol. 10(2); 93-95, 2017.

\begin{abstract}
Nowadays, a dental implant is considered a good alternative to replace the missing teeth. However, there are several requirements needed for the implant placements and for a good resolution. Present studies demonstrate that the immediate implant placement posterior to the extraction of the tooth obtains better long-term results and also preserves the natural alveolar socket. This facilitates the placement and correct adjustment of the dental implant, only if the extraction is done atraumatically and without complications. Therefore, the present case reports a 73-year-old male patient with arterial hypertension and type II diabetes mellitus background, controlled by his medical doctor. An atraumatic tooth extraction of OD\#46 was carried out on this patient, with immediate dental implant NORMON HI placement and insertion of xenograft bone BIO-GEN in order to ensure correct osseointegration. This procedure was done without complications and with correct position and parallelism.
\end{abstract}

\section{KEY WORDS}

Dental implants, diabetes mellitus, systemic diseases, tooth extraction.

Rev. Clin. Periodoncia Implantol. Rehabil. Oral Vol. 10(2); 93-95, 2017.

\section{INTRODUCCIÓN}

Hoy en día son múltiples las razones por las cuales una persona pierde órganos dentarios, inicialmente debido a falta de cuidado de ellos y la no evaluación periódica con el odontólogo. Sin embargo, son diversas las enfermedades sistémicas que también alteran y agravan ciertos componentes de la cavidad oral, entre los que se encuentran la diabetes mellitus, por lo cual conlleva finalmente a la pérdida de ellos si no se tratan con anterioridad. ${ }^{(1)}$ Por ende, debido a que todo diente permanente perdido no vuelve a erupcionar ni desarrollarse, se han creado nuevos materiales e instrumentos para reemplazar estas piezas dentarías faltantes en la cavidad oral; por tal razón, se empezaron a utilizar los implantes dentales, como medida alterna para recrear artificialmente lo que sería un órgano dentario. ${ }^{(2)}$ Cabe resaltar que para la colocación de estos se tienen en cuenta diferentes factores como: altura y espesor de hueso, espacio disponible para la colocación de éste, edad del paciente paciente sistémicamente controlado, correcta cicatrización, entre otros. ${ }^{(3)}$

Según la literatura científica se establece que los implantes dentales en personas sanas presentan rangos de éxito entre el 90 y $95 \%$ a los 10 años posterior a su colocación. En cambio, en pacientes sistémicamente comprometidos existen mayores riesgos con respecto al éxito en la colocación de implantes y a largo plazo también puede verse afectado por alguna alteración en el organismo. ${ }^{(4)}$

La diabetes mellitus además, es la patología sistémica más común en las personas y está estipulado que tiene ciertas contraindicaciones relativas pero no absolutas, con respecto a los implantes dentales. (1) Si bien es cierto, las personas que padecen de diabetes están más predispuestas a tener pérdida de órganos dentarios que las no-diabéticas. Claro está, debido a que una alteración en el calcio y fósforo, esta puede manipular una correcta remodelación y mineralización de hueso, por 
lo tanto el paciente diabético debe mantenerse controlado, con niveles de glucosa sanguíneos estables para proceder a colocar un implante dental y así reducir los riesgos a corto y largo plazo, y obtener mejores resultados con respecto a oseointegración y buena captación de este en el hueso alveolar. ${ }^{(1)}$

Ahora bien, existe una nueva clasificación según el tiempo de colocación del implante dental (5) la cual está organizada de la siguiente manera: Tipo 1: Implante dental colocado inmediatamente luego de la extracción dental, sin curación de hueso ni tejidos blandos. Tipo 2: Colocación temprana del implante dental, aproximadamente 4 a 8 semanas después del proceso de curación, con algunos tejidos blandos cicatrizados y el sitio post extracción presenta tejido blando cicatrizado cubriendo el alveolo pero sin cicatrización ósea significante. Tipo 3: Colocación temprana del implante dental con cicatrización ósea parcial, aproximadamente 12 a 16 semanas de curación; el sitio post extracción presenta cicatrización de tejidos blandos y curación ósea significante. Tipo 4: Colocación tardía del implante dental, un poco más de 6 meses después de la extracción dental. Implante dental colocado en un sitio edéntulo completamente cicatrizado.

Por consiguiente, de acuerdo a esto, estudios recientes han encontrado que resulta mejor colocar el implante dental inmediatamente después de realizar la extracción dental del órgano dentario afectado (tipo 1) para así aprovechar el alveolo dental ya moldeado y evitar la reintervención quirúrgica del paciente. ${ }^{(2)}$ Además, en la literatura científica existen casos reportados que demuestran que el implante dental inmediato presenta mejor remodelación y relleno óseo que el colocado tiempo después de la extracción y proceso de cicatrización. ${ }^{(3)}$ Además que el implante dental inmediato posee ciertas ventajas como lo son menos intervenciones quirúrgicas, someter al paciente a fármacos en una sola ocasión. Sin embargo, en diveras situaciones hay que recurrir al uso de materiales regenerativos o injertos óseos para lograr una mejor estabilidad. ${ }^{(6)}$ Por tal razón, en este artículo se reporta el caso clínico de un paciente controlado al cual se le realizó implante dental inmediato.

\section{REPORTE DE CASO CLÍNICO}

Paciente de sexo masculino de 73 años de edad, el cual acude a consulta odontológica debido a que presenta un molar extruido; entre sus antecedentes sistémicos personales se encuentra hipertensión arterial, diabetes mellitus tipo II, bronquiectasia, gastritis y cálculos renales. Refiere estar controlado con su médico tratante, por lo cual ingiere Metformina hidrocloruro $850 \mathrm{mg} /$ día y Glicazida $30 \mathrm{mg} /$ día para la diabetes, Doxazosina mesilato $8 \mathrm{mg} /$ día para la HTA y Omeprazol 20mg/ día para la gastritis. Al examen clínico intraoral se observaron recesiones gingivales y bolsas periodontales de hasta $4 \mathrm{~mm}$ no activas, el OD\#46 fracturado en porción distal y OD\#45 fracturado en porción MOD con presencia de amalgama, por lo cual se procedió a obturar el órgano dentario con resina. Se indicó la extracción del OD\#46, con colocación inmediata de implante dental; por tanto se procedió a realizar profilaxis dental en primera instancia y se hizo interconsulta con el médico tratante, el cual autorizó realizar el procedimiento sin ningún problema debido a que el paciente se encontraba sistémicamente controlado. Se ordenaron exámenes paraclínicos de glicemia en ayunas y hemoglobina glicosilada, el cual arrojó resultados normales.

Inicialmente se tomó la presión arterial del paciente, la cual estaba en 120/80 $\mathrm{mmHg}$. Se le realizó profilaxis antibiótica con Amoxicilina 1g una hora antes del procedimiento. Se inició la extracción atraumática del OD\#46 con previa colocación de anestesia troncular mandibular con Ultracain con epinefrina $40 / 0,005 \mathrm{mg} / \mathrm{ml}$ y anestesia infiltrativa con mepivacaína NORMON 3\%. Después se realizó una incisión supracrestal intrasulcular alrededor del OD\#44 y OD\#45 con descarga hacia lingual y vestibular, con hoja de bisturí n. 12. Luego se desprendió el colgajo con periostótomo y se procedió a iniciar la extracción. Se inició la sindesmotomía, y se realizó una odontosección con fresa de carburo de tungsteno n.703, se hizo luxación con elevador angulado y se extrajo la raíz distal y luego mesial del respectivo diente.

Inmediatamente después de realizar la extracción dental se procedió a insertar el implante dental. Se comenzó con fresa lanceolada para marcar el punto donde se colocará el implante, el cual fue justo en el tabique alveolar indicando que era el sitio medio donde se ubicaba el órgano dentario. (7) Luego, se utilizó una fresa de diámetro $2 \mathrm{~mm}$, fresa piloto de diámetro 2-3,1 $\mathrm{mm}$, fresa de diámetro $3.1 \mathrm{~mm}$, fresa piloto de diámetro $3.1-4.1 \mathrm{~mm}$, fresa de diámetro $4.1 \mathrm{~mm}$ y por ultimo fresa de perfil de diámetro 4,75 de conexión interna, respectivamente, para así por último utilizar una fresa de terraja. Así, se procedió a colocar el implante dental NORMON HI de 4.75 de diámetro por $10 \mathrm{~mm}$ de longitud, el cual quedó a $2 \mathrm{~mm}$ en sentido vertical con respecto al margen oseo de la tabla cortical. (Ver Fig. 1) Después se colocó xenoinjerto óseo BIO-GEN bovino particulado, el cual se compactó ligeramente con un instrumento bola huevo, para no afectar la vascularización; finalmente se hizo sutura simple, colchonero y en cruz. Se recetó Augmentine 500mgs (amoxicilina con ácido clavulánico) cada 8 horas durante 7 días, paracetamol $1 \mathrm{~g}$ cada 8 horas durante 4 días y Perio Aid Clorhexidina $0,12 \%, 10 \mathrm{ml}$ cada 12 horas por 15 días. El procedimiento finalizó sin complicaciones y la ortopantomografía final evidencia la correcta colocación del implante dental, con buen paralelismo, mediante el uso de referencias intrabucales como el OD\# 45, y adecuada distancia entre diente-implante, de $1.5 \mathrm{~mm}$ en sentido horizontal (Ver Fig. 2)

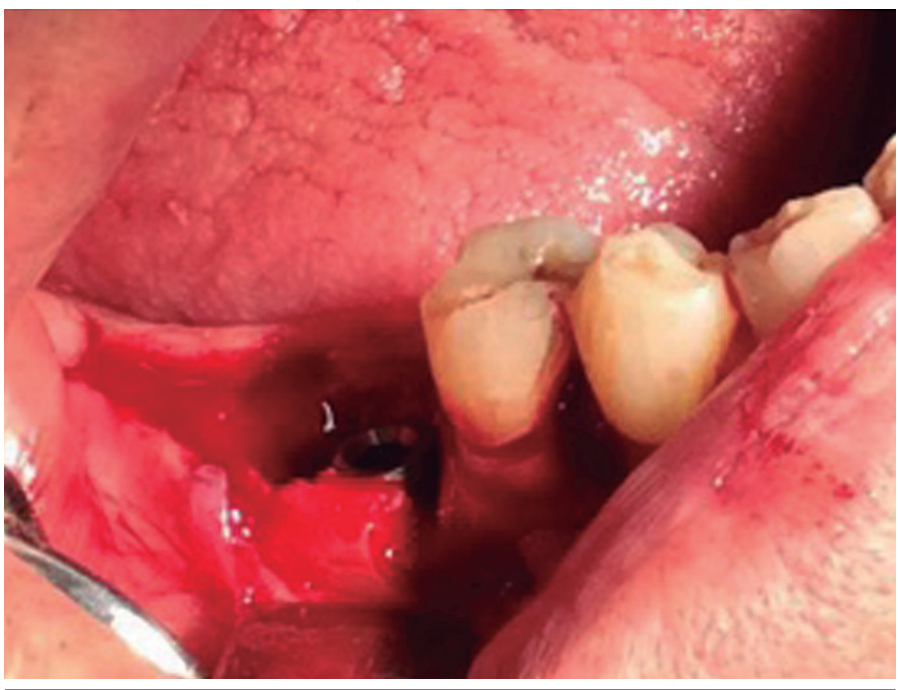

Figura 1. Implante dental NORMON HI inmediato después de extracción dental de OD 46; se observa además el espacio alveolar restante alrededor del implante.

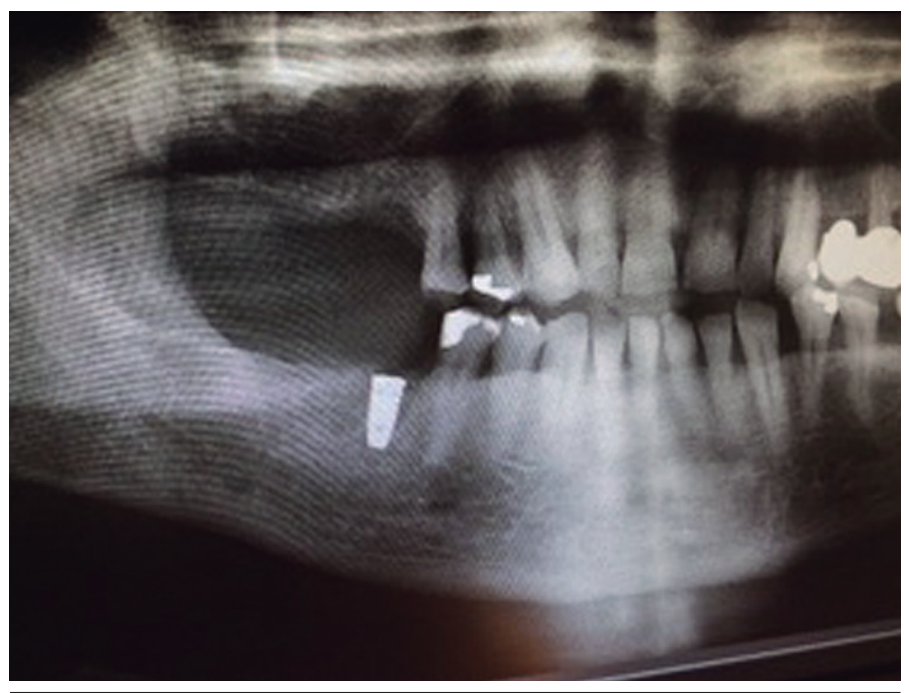

Figura 2. Ortopantomografía final que muestra la correcta posición del implante dental NORMON HI con adecuado paralelismo y distancia con el OD\#45.

\section{DISCUSIÓN}

El éxito de un implante dental en pacientes sistémicamente comprometidos está en un $90-95 \%$ en un tiempo de 10 años. Sin embargo, existen estudios que demuestran la pérdida del implante dental debido a falta de oseointegración en el proceso de curación o infección de los tejidos peri-implantares. ${ }^{(4)}$ Recientes estudios han encontrado resultados similares con respecto al éxito de un implante dental en pacientes diabéticos controlados y en pacientes no-diabéticos, por lo cual siempre y cuando el paciente mantenga un tratamiento adecuado y un buen seguimiento de su glicemia en sangre, se podrían realizar los procedimientos sin ningún problema. ${ }^{(1,8)}$, hay que asegurarse también que el paciente continúe ingiriendo los medicamentos después del procedimiento. ${ }^{(4)}$

Ahora bien, hay casos que reportan resultados favorables de implantes dental inmediatos sin necesidad de colocar materiales regenerativos ${ }^{(3)}$ Sin embargo, Sanz et al en el 2012, mediante una revisión sistemática 
encontraron que el aumento de hueso y el uso de autoinjertos óseo o membranas reabsorbibles utilizadas conjuntamente con la colocación de implantes dentales poseen mejores resultados a largo plazo, lo cual complementa con la literatura encontrada anteriormente. ${ }^{(5)}$

Adicionalmente, Lanza et al. en 2015 mencionan que después de una pérdida dental, ya sea mediante extracción o sin intervención, siempre sucederá una pérdida ósea por lo que la colocación inmediata del implante dental no podrá detener este fenómeno fisiológico; por ende, la utilización en conjunto de materiales regenerativos o autoinjertos óseos con los implantes dentales inmediatos resulta efectivo para prevenir en mayor cantidad la pérdida ósea horizontal o vertical que podría suceder después. ${ }^{(9)}$, lo cual concuerda con el procedimiento y protocolo realizado en el presente caso.

Hay que tener en cuenta también, que la diabetes mellitus presenta altas tasas de fracaso debido a la falta de oseointegración que ocurre y la presencia de infecciones, pero según Gómez de Diego et al. en el 2014 esto puede preverse y solo ser un riesgo relativo con respecto a la colocación de implantes dentales si el paciente se encuentra controlado y sea recetado con antibióticos previo al procedimiento y técnicas de asepsia con enjuagues de clorhexidina al $0,12 \%{ }^{(10)}$, el cual fue realizado con el paciente del presente caso, por lo que se espera obtener resultados favorables y exitosos a largo plazo.

Cabe destacar que no solo las patologías de base intervienen en el éxito del implante dental sino que existen también otros factores que predisponen o manipulan esto. Tomasi et al. en 2010, mediante un modelo multinivel encontró que la edad y fumar son factores de importancia con respecto a la colocación de implantes dentales. ${ }^{(11)}$ Según resultados encontrados, a mayor edad que presenta el paciente, hay una disminución en la condrogénesis, reducida formación ósea y vascularización, retrasada remodelación y una alteración en la expresión de los genes que intervienen en la remodelación y reparación. ${ }^{(11)}$ Por lo tanto, debido a que el paciente del presente caso también era mayor de edad, con 73 años, también había que tener en cuenta este factor junto con la diabetes mellitus y el tipo de hueso alveolar que presentaba, por lo cual se le suministró el xenoinjerto óseo junto con el implante dental inmediato.

En conclusión, la colocación de implantes dentales en pacientes sistémicamente comprometidos no debe considerarse como una contraindicación si el paciente se encuentra controlado con su médico especialista, y se sigan las medidas necesarias para evitar cualquier respuesta desfavorable, realizando profilaxis antibiótica, medidas asépticas y antisépticas, exámenes paraclínicos y medicamentos postoperatorios para evitar inflamación e infección. (8) Además, al realizar implantes dentales inmediatos se evita correr el riesgo doble de infección o de manipulación de los tejidos, en donde nos referimos a que se evita doble infección puesto que solo se está interviniendo quirúrgicamente al paciente una sola vez; además de que presenta casi los mismos resultados de éxito con respecto a los implantes colocados después de la curación del alveolo dental. ${ }^{(6,12,13)}$ Pero, esta técnica quirúrgica no está exenta de complicaciones, entre las cuales la más común es la presencia de infección postoperatoria que puede inducir a la pérdida del implante dental, por esta razón se deben realizar todas las medidas e indicaciones necesarias para colocar el implante inmediato.

\section{AGRADECIMIENTOS}

El presente caso y sus autores agradecen al laboratorio dental NORMON S.A por su contribución con el implante dental NORMON HI y el autoinjerto óseo BIO-GEN, además de la contribución del Master de Odontología Integrada al Adulto y Paciente Especial, de la Universidad de Sevilla.

\section{Bibliografía}

1. Inbarajan A, Veeravalli PT, Vaidyanathan AK, Grover M. Short-term evaluation of dental implants in a diabetic population: an in vivo study. J Adv Prosthodont. 2012;4(3):134-8.

2. Sanz I, Garcia-Gargallo M, Herrera D, Martin C, Figuero E, Sanz M. Surgical protocols for early implant placement in post-extraction sockets: a systematic review. Clin Oral Impl Res. 2012;23(s5):67-79.

3. Moghe S, Moghe A, Shah R, Pillai A, Nahar S, Goyal N. Immediate Implantation into extraction socket, a case report. IOSR-JDMS. 2014;13(7):59-63.

4. Diz P, Scully C, Sanz M. Dental implants in the medically compromised patient. J Dent. 2013:41(3):195-206.

5. Lang NP, Pun L, Lau KY, Li KY, Wong MC. A systematic review on survival and success rates of implants placed immediately into fresh extraction sockets after at least 1 year. Clin Oral Impl Res. 2012;23(s5):39-66.

6. Ripollés de Ramón J, Gómez Font R, Bascones-llundain C, Bascones-llundain $\mathrm{J}$, Bascones-Martínez A. Estudio clínico de los implantes dentales Naturactis en postextracción dental. Av Periodon Implantol. 2013;25(3):135-42.

7. Telletxea O, Sánchez E, Iturre B, Aguirre LA, Velilla JR, Estefanía E. Implantes inmediatos post-extracción: revisión de la literatura. Clínica Periodontal. 2011; 21(2):123-35.
8. Dubey RK, Gupta DK, Singh AK. Dental implant survival in diabetic patients; review and recommendations. Natl J Maxillofac Surg. 2013;4(2):142-50

9. Lanza A, Scognamiglio F, Femiano F, Lanza M. Immediate, early, and conventional implant placement in a patient with history of periodontitis. Case Rep Dent. 2015;2015:217895

10.Gómez-de Diego R, Mang-de la Rosa Mdel R, Romero-Pérez MJ, CutandoSoriano A, López-Valverde-Centeno A. Indications and contraindications of dental implants in medically compromised patients: Update. Med Oral Patol Oral Cir Bucal. 2014;19(5):e483-9

11.Tomasi C, Sanz M, Cecchinato D, Pjetursson B, Ferrus J, Lang NP, et al. Bone dimensional variations at implants placed in fresh extraction sockets: a multilevel multivariate analysis. Clin. Oral Impl. Res. 2010;21(1):30-6.

12.Rodrigo D, Martin C, Sanz M. Biological complications and peri-implant clinical and radiographic changes at immediately placed dental implants. A prospective 5-year cohort study. Clin. Oral Impl. Res. 2012;23(10):1224-31.

13. Covani U, Cornelini R, Louis Calvo J, Tonelli P, Barone A. Bone remodeling around implants placed in fresh extraction sockets. Int J Periodontics Restorative Dent. 2010;30(6):601-7. 